\title{
Germanica
}

\section{Visages du fantastique dans les lettres scandinaves modernes}

Aspekte der Phantastik in der modernen skandinavischen Literatur

\section{Régis Boyer}

\section{OpenEdition}

12 Journals

Édition électronique

URL : http://journals.openedition.org/germanica/2826

DOI : $10.4000 /$ germanica.2826

ISSN : 2107-0784

Éditeur

Université de Lille

\section{Édition imprimée}

Date de publication : 30 juin 1988

Pagination : 131 à 149

ISSN : 0984-2632

\section{Référence électronique}

Régis Boyer, «Visages du fantastique dans les lettres scandinaves modernes », Germanica [En ligne],

3 | 1988, mis en ligne le 10 septembre 2015, consulté le 06 octobre 2020. URL : http://

journals.openedition.org/germanica/2826; DOI : https://doi.org/10.4000/germanica.2826

Ce document a été généré automatiquement le 6 octobre 2020.

(C) Tous droits réservés 


\title{
Visages du fantastique dans les lettres scandinaves modernes
}

\author{
Aspekte der Phantastik in der modernen skandinavischen Literatur
}

Régis Boyer

1 Les considérations que voici sont inspirées par une constatation, en soi surprenante a priori, qu'il m'est arrivé de faire depuis bien longtemps et qu'une longue fréquentation des textes eddiques, scaldiques ou de sagas n'a jamais démentie: le Nord ancien connaissait bien peu de "monstres", lesquels, de toute manière, ne supportent simplement pas la comparaison avec ceux qui hantent à foison les littératures anciennes grecque, latine, védique, slave ou celtique. Un ours à trois têtes ici, un Grand Serpent de Midgardr ou un Loup / Chien gigantesque là, un dragon ailleurs, c'est finalement bien peu de chose en regard de ce que peut fournir, chose plus étonnante encore, la littérature germanique continentale, sous ce rapport, telle qu'elle a été magistralement étudiée par Claude Lecouteux, par exemple ${ }^{1}$. Même sans s'égarer dans de subtiles et souvent spécieuses distinctions entre fabuleux, imaginaire, irréel, merveilleux, surnaturel, bizarre, extraordinaire, etc..., il est clair que, dès les origines connues, le monde mental nord-germanique n'a pas privilégié l'imagination ou la fantaisie et que, quoique très familier d'une sorte d'osmose permanente avec l'au-delà (mort et vif pouvant, d'aventure, entrer presque spontanément en contacts réciproques ${ }^{2}$ ), il ne s'est guère égaré hors du possible ou du réel. Une étude approfondie du conte populaire scandinave, avec ses trolls, tomtar, rå, nisses ou nixes, houldres, et bergfolk ne passe pas la démesure non plus et même quand, dans la ligne de ce type d'inspiration, H.C. Andersen met en scène sa petite sirène ou Selma Lagerlöf son nain Nils Holgersson, nous ne saurions prétendre évoluer dans le «fantastique » qu'à titre de référence lointaine, tant ces auteurs - et leurs émules - s'attachent à " humaniser " et à "dédramatiser» ce que leur sujet pourrait avoir d'excessif ou de carrément fantasmatique.

2 Évidemment, ce genre d'approche exige une définition claire du fantastique et, comme on le sait, il n'en manque pas ${ }^{3}$. Je m'en tiendrai à celle, très simple, que propose Roger Caillois dans la préface de son Anthologie du Fantastique (Club Français du Livre, 1958, 
p. 3) - où, notons ce point, il identifie fantastique et terreur et où il ne cite simplement aucun texte scandinave moderne, alors qu'il fait une place de choix, pour m'en tenir aux peuples " germaniques ", à l'Angleterre, aux États-Unis, à l'Allemagne et aux Flandres : «la terreur doit être engendrée seulement par une intervention surnaturelle; l'intervention du surnaturel doit obligatoirement aboutir à un effet de terreur ». Cette approche élimine, je le sais bien, bon nombre de connotations d'un terme par excellence polysémique, mais elle a l'avantage de cerner le sujet en son essence même, me semble-t-il, dans la mesure ou, pour continuer de citer Caillois, «le fantastique manifeste un scandale, une déchirure, une irruption insolite, presque insupportable dans le monde réel » (loc. cit.), ou encore : « le fantastique suppose la solidité du monde réel, mais pour mieux la ravager ». C'est pourquoi j'écarte d'emblée du sujet tout ce qui ressortirait à la science-fiction, genre qui n'a pas laissé indifférents, quoique avec modération, les Scandinaves : je pense à Kallocain de Karin Boye ou à Epp d'Axel Jensen ; tout comme je ne retiens pas un certain type de fantastique non effrayant, ce qui ne signifie pas qu'il ne soit pas intéressant, pour la psychanalyse notamment, comme celui qui préside aux faits et gestes de la Pippi Långstrump d'Astrid Lindgren.

Autant que je puisse voir, une petite étude du genre de celle que je propose ici pourrait apporter une contribution utile à l'analyse ethnopsychologique des Scandinaves à travers leurs littératures. C'est donc sous cet angle que je propose de l'aborder.

La méthode sera très simple, elle aussi, et sera de nouveau empruntée à Roger Caillois, orfèvre en la matière, l'application étant faite aux littératures scandinaves modernes, sans souci d'exhaustivité s'il faut le dire.

Or, dans une étude passionnante, R. Caillois (article "Fantastique» de l'Encyclopaedia Universalis) propose de distinguer douze variantes thématiques du fantastique. Je vais les passer en revue, en cherchant s'il se trouve des cas où la correspondance exacte se rencontre du côté de la Scandinavie. J'entends bien que, conformément à l'orientation générale du présent numéro de cette revue, il s'agira de "marges du fantastique " puisque, sauf rarissimes exceptions - je pense à Karen Blixen, et encore, dans une partie seulement de son œuvre - je ne vois pas d'écrivain scandinave dont toute la production ait été placée sous le signe du fantastique. Il est d'ailleurs tout à fait instructif qu'un ouvrage de référence, alphabétique et analytique, comme le Svenskt Litteraturlexikon (Gleerup Lund $2^{\mathrm{e}}$ ed., 1970) n'ait simplement aucune entrée «Fantastisk Litteratur», alors qu'il en a une, par exemple, pour «Fabel» ou une autre pour «Folksagn». Je n'entends pourtant pas insinuer par là que le fantastique, en tant que catégorie littéraire, est totalement étranger à la mentalité scandinave, mais, pour anticiper sur mes conclusions, qu'il faut sans doute le chercher ailleurs que dans la panoplie reçue du genre. Donc, partant du principe, très respectable, qu'il n'y a pas de fantastique sans collusion, d'une manière ou d'une autre, avec l'Autre monde, Caillois propose la classification thématique suivante :

6 1) Pactes avec le Démon (type Faust). Sans remonter à des sources très lointaines qui pourraient, d'ailleurs, fort bien être dues elles-mêmes à des influences chrétiennes voilées - je pense à une saga islandaise comme Víga-Glúms saga où il semble bien que s'affrontent, sans que cela nous soit explicitement dit, à l'islandaise, deux clans dont 
l'un a pactisé avec Ódinn, l'autre, avec Freyr :on sait que l'Église s'est attachée à ravaler les anciens dieux païens au rang de diables - le motif a été tout aussi populaire dans le Nord qu'ailleurs. Le Gösta Berling de Selma Lagerlöf, elle-même plus nettement préoccupée de fantastique que bon nombre de ses confrères, ou Le Voyage de Jonathan (1941) du Danois Martin A. Hansen nous présentent des personnages (Gösta, Jonathan) qui ont bien fait une sorte de pacte, à des fins précises, avec le Diable, lequel figure du reste, expressément, chez Hansen, sous les espèces du valet roux Askelad (la rousseur se souvient du dieu Thórr, figure courante du Diable dans les ballades populaires, folkeviser, ou les contes, folkesegn, scandinaves). Il est remarquable que le seul écrivain islandais moderne que l'on puisse tenir pour un très grand dramaturge, Johann Sigurjónsson se soit imposé par deux pièces dont la seconde, Galdra-Loftur (Loftur le Magicien, 1915) reprend exactement le thème goethéen, aux mêmes fins : séduction et puissance. Il n'y a pas lieu de développer. Nous sommes ici dans la thématique des grands types universels de toutes nos littératures et l'on ne voit pas pourquoi les Scandinaves n'y auraient pas sacrifié. On fera remarquer que les modèles proposés n'ont tout de même pas les dimensions métaphysiques de Faust. En vérité, comme je l'ai déjà dit, les Scandinaves ne se trouvent peut-être pas tellement tentés par ce genre d'argument, c'est plutôt le prétexte d'affabulation qui aura séduit les quelques auteurs que je viens d'évoquer. Mais, Dieu ou Diable, les visionnaires que restent volontiers les descendants de Sainte Brigitte ou de Swedenborg, ou des auteurs inconnus de la Völuspá islandaise ou du Draumkvaedi norvégien (respectivement vers 1000 et 1200) n'ont pas dû trouver tellement étonnant ce genre de pacte qui, au demeurant, recoupait parfaitement ce que nous pouvons savoir du type de relations qu'entretenait, sur le mode "donnant-donnant », le zélateur du paganisme ancien et son dieu préféré ou fulltrúi ${ }^{4}$.

7 2) Le second motif concerne « l'âme en peine qui exige pour son repos qu'une certaine action soit accomplie : un défunt revient sur terre pour persécuter son meurtrier ; un châtiment attache un fantôme au lieu où il a accompli son forfait ». Caillois note que ce type de revenant était connu de l'antiquité grecque. Il aurait pu ajouter qu'il est monnaie courante, au point de constituer, dans les sagas islandaises, un véritable type littéraire, dans l'antiquité scandinave. C'est le personnage du draugr, un mort-malmort, soit parce qu'il n'a pas été inhumé selon les rites requis, soit, en effet, parce que son assassin n'a pas été découvert ou châtié selon les règles, soit encore parce que sa descendance n'a pas su faire fructifier le patrimoine à son goût $t^{5}$. Les sagas, qui en connaissent un nombre impressionnant, nous en proposent au moins deux de premier ordre : le Thorbjörn l'Estropié d'Eyrbyggja saga (La Saga de Snorri le godi) et le Glámr de Grettis saga ${ }^{6}$. Forts de telles assises, il n'est pas surprenant que ce personnage hante durablement les lettres scandinaves, parfois sous des formes extrêmement subtiles qui reviennent à des variantes de métempsycose ou de réincarnation, comme dans l'admirable nouvelle de Sven Delhlanc intitulée «La femme du pasteur " (et qui est jointe en annexe à cette étude) (1970). Le draugr, sous une forme encore plus proche de la définition de Caillois, l'útilegumadr, c'est-à-dire, effectivement, un condamné qui doit errer comme une âme en peine, en compagnie de ses semblables - est une figure finalement assez voisine de la Chasse Sauvage ou Mesnie Hellequin qui hante littéralement le folklore, notamment norvégien. Le grand romantique islandais Matthías Jóchumsson mettait en scène des útilegumenn dans le drame qui porte ce titre (1864) et l'on se sent fondé à dire que, toute théories scientistes mises à part, l'attraction qu'exerça le thème des tares héréditaires, du passé qui nous suit 
inexorablement, par exemple sur Ibsen dans ses Revenants (1881) ressortit finalement à la même analyse. En un sens, et surtout dans la mesure, très grande en Scandinavie, où un écrivain se fait mémorialiste, il n'est pas beaucoup de grandes œuvres qui n'aient été marquées de ce thème : prenez, par exemple, le Suédois Lars Gyllensten, avec la série qui s'ouvre par Juvenilia (1965). À tout propos, le draugr, sous quelque travesti que ce soit, resurgit, serait-ce, tout naturellement, au détour d'un recueil de nouvelles de Knut Hamsun, Kratskog (1093) où " Un fantôme », sur une sombre histoire de dent volée à un cadavre, renoue avec la grande tradition islandaise. Et d'ailleurs, faut-il chercher plus loin la prédilection que manifestent ces littératures volontiers « noires » pour les cimetières, la mort et ses affres, et que traduit si bien dans ses répétitions obsessionnelles l'œuvre picturale du Norvégien Edvard Munch? Un grand roman picaresque, truculent et haut en couleur comme La Cloche d'Islande de H. Laxness ne se refuse pas le visible plaisir d'une frénétique danse macabre à laquelle est mêlé le héros, Jón Hreggvidsson, au chapitre intitulé «L'aventure allemande » (1943). Et j'ai à peine besoin de rappeler que le draugr vit dans une si grande intimité avec l'inspiration de la Danoise Karen Blixen qu'on le sent présent, non seulement dans la majorité de ses Contes, mais même au détour de certains de ses Essais.

8 3) Le troisième type est, au fond, une variante particulièrement célèbre du précédent: c'est le Juif Errant condamné à traîner lamentablement une existence maudite jusqu'à la consommation des temps. L'insistance, ici, est à peine nécessaire. Faut-il rappeler qu'avec Don Juan et Faust, c'est un des trois grands types symboliques qui, pour Kierkegaard, incarnent les "étapes sur le chemin de la vie ", en l'occurrence, le stade éthique. Il a hanté les consciences scandinaves, ne serait-ce qu'en vertu des réflexions qui ont été avancées au dernier paragraphe. Il n'y a donc pas à s'étonner de le trouver au premier plan d'une œuvre qui, de toute façon, vit en grande connivence avec l'audelà, avec «Le pays des âmes » dont il est question dans Âmes masquées (1930), celle du Suédois Pär Lagerkvist, puisque la série de ses « récits de pèlerin » s'ouvre précisément par La mort d'Ahasverus (1960). Mais, là encore, il convient de souligner que ce n'est pas tellement l'aspect "fantastique», au sens où, R. Caillois nous l'a dit, la terreur se conjugue par définition au récit, qui prédomine chez ce personnage tel que le voient les Scandinaves. Il est le grand mort-mal-mort parce que, dans une perspective que n'aura pas manqué de renforcer la dialectique bien luthérienne de la faute et de l'expiation (mais il faut préciser qu'en ce domaine-là aussi, le terrain était hautement favorable : tous les textes de lois les plus anciens se fondent à peu près exclusivement sur le jeu de la «brèche » commise dans la réputation ou l'honneur, ou l'intégrité familiale, ou la fortune de l'offensé, et de la "compensation" dûment codifiée qui la comblera) il a gravement manqué à la Loi, ou, sous un autre angle, a trop intimement vécu dans le commerce du Grand Secret. Le Danois Johannes Jørgensen voyait, au demeurant, Ahasverus dans une lumière différente: dans Confession (1894), recueil poétique qui revient à une intense méditation sur l'étrangeté de la condition humaine ici-bas, Ahasverus est un symbole de notre inlassable quête d'éternité : c'est-à-dire qu'il a complètement dépouillé tout aspect fantastique ou, plus exactement, dans une perspective qui, assurément, nous met aux sources mais dépasse de bien loin le propos «terrifiant» dont part Caillois, qu'il a rejoint la dimension la plus profonde de cette notion, celle qui lui vaut d'échapper totalement aux capacités de notre imagination. Je ne trouve pas fortuit que le seul vraiment très grand philosophe scandinave, un des plus grands mystiques danois et l'écrivain suédois dont j'ai essayé de démontrer ailleurs ${ }^{7}$ que toute entreprise se ramenait finalement à une quête mystique se soient 
involontairement rejoints autour du personnage d'Ahasverus. Il m'a toujours paru que la conscience littéraire scandinave, est-ce fréquentation constante de l'étrange et transfiguratrice lumière du Nord, est-ce connivence naturelle avec la pureté inhumaine de la glace et du froid, vivait de plain-pied avec une manière d'aperception instinctive de l'infini, ou, au moins, de l'indéfini qui est, en soi, une exaspération du fantastique dans la mesure, encore une fois, où celui-ci manifeste les impuissances de l'imagination ou l'inachèvement du réel. J'irai même plus loin : si tant de ces œuvres, de Swedenborg à Dagerman en passant par Almquist, s'acharnent tant à découvrir l'équation, comme mathématique, qui parviendrait enfin (entreprise vaine, s'il faut le dire) à rendre compte rationnellement du réel, n'est-ce pas parce qu'elles se trouvent coïncider avec l'essence même du personnage d'Ahasverus? Il y a un réflexe que je dis proprement fantastique dans la notation tant de fois citée, de Barabbas, chez Lagerkvist, rendant l'esprit dans un dernier cri « dans l'obscurité, comme si c'était à elle qu'il s'adressait ». L'errance fut vaine et ne pouvait avoir d'autre fin qu'elle-même.

9 4) Et c'est encore la même idée qui préside à « la mort personnifiée apparaissant au milieu des vivants ». Ici, bien entendu, c'est au Septième Sceau d'Ingmar Bergman que l'on songe immédiatement. Je dirai tout de même que, pour surprenant que ce soit, le thème n'est finalement, pas tellement présent dans les lettres scandinaves, même si une nouvelle de J.-P. Jacobsen, comme «La Peste à Bergame » (1886) ou un récit comme Le Charretier de la Mort de Selma Lagerlöf (1912) l'orchestrent eu majeur. C'est que, je viens de le dire, ces écrivains comptent, naturellement si l'on peut s'exprimer ainsi, la Mort parmi leurs personnages, soit ouvertement, soit selon une ambiguïté souveraine comme chez Strindberg où, surtout à partir de ses pièces oniriques, elle est constamment présente, telle quelle (La danse de Mort, 1901) ou dans une «fantastique » collusion avec la Femme (Le Chemin de Damas, 1898-1904), collusion dont il y aurait tant à dire que cela déborderait vraiment trop le cadre de cette petite étude : rappelleronsnous, pourtant, que l'un des principaux visages de la Grande-Déesse (ou Déesse-Mère, ou Terre-Mère) que connut la mythologie scandinave antique convient à la Mort (Skadi, Hel) ? Celle qui fut la véritable initiative du modernisme dans le Nord, la SuécoFinlandaise Edith Södergran qu'une destinée tragique allait faire mourir prématurément, l'aura dit mieux que personne dans un splendide poème adressé à la lune dont, peut-être, elle ne savait pas qu'elle présidait précisément à la mort dans l'antiquité païenne :

Vad allting som är död är underbart

Och outsägligt,

Ett dött blad och en död människa

Och måtnens skiva ${ }^{8}$

10 Il arrive, comme je l'ai dit, que l'on juge "morbides » les écrivains du Nord parce qu'incontestablement, leur inspiration est souvent « noire »- je pense, pour ne prendre qu'un exemple actuel et parce qu'elle est relativement bien connue en France, à Brigitta Trotzig. Soit! Disons qu'ils ont, mieux que leurs confrères dans d'autres cultures, intégré cette dimension à leur univers. Entreprise ou état de faits réellement fantastique, mais bien au delà des petites affabulations datées, classées et destinées à faire peur...

11 5) C'est pourquoi la catégorie suivante, sans être négligée, est pourtant, je crois, assez peu représentée en Scandinavie. Il s'agit de « la chose » indéfinissable et invisible [...] qui tue ou qui nuit ", le modèle inégalé en étant Le Horta de Maupassant. En vérité, je n'en vois pas d'équivalent strictement exact dans le Nord (sauf en Finlande, chez 
Linnankoski par exemple, qui sort du cadre de cette ar1alyse). À moins qu'on ne pressente, dans le roman riche et complexe du Norvégien Tarjei Vesaas, L'Incendie (1961), cette « chose » qui brûle, tue, désole ou rend fou. Sans doute, ici, la psychanalyse aurait-elle son mot à dire, dans une acception bien bachelardienne : c'est le regard du héros, Jon, qui est incendiaire, tout comme dans une nouvelle de l'« absurdiste » danois Villy Sørensen, «L'ennemi » (dans Histoires anodines, 1955). Vesaas me fournit d'ailleurs l'occasion bienvenue d'un petit développement encore sur le sujet qui nous préoccupe. Apparemment, rien n'est moins « fantastique » que cette œuvre lourde et lente, pleine de simples d'esprit (Les Oiseaux, 1957), de petites filles (Palais de Glace, 1963) ou même... de porcs (Le Germe, 1940), le tout dans un décor de son Trøndelag, bien rural, bien quotidien, osons même dire bien banal. Et pourtant, la réalité n'est jamais ce qu'il nous en donne à voir'. René Char eût dit qu'elle est constamment soulevée. La Maison dans les ténèbres (1945), avec ses personnages allégoriques et sa forêt de symboles, va bien plus loin qu'une simple évocation de la Norvège sous l'occupation allemande. Pour un peu, je dirais que la simplicité et le réalisme y sont si bruts qu'ils en deviennent fantastiques. Et je devine que c'est la Mort, du reste plus ou moins personnifiée dans L'Incendie, perpétuellement présente à l'arrière-plan des consciences, qui métamorphose ces banales (en apparence) histoires en dialogues profonds avec la puissance des ténèbres. Les Scandinaves ne sont jamais aussi grands que lorsqu'ils s'attachent, avec leurs voix de conteurs inlassables, à manifester l'évidence de l'indissoluble union que disent toutes leurs belles histoires d'amour et de mort. Le sensationnel ou le directement caractérisé ne sont pas nécessairement au rendez-vous : entendons que la Mort en tant que personnage à part entière n'y est pas toujours immédiatement repérable. Mais présente, elle l'est. Les beaux récits cosmopolites de l'Islandais Thór Vilhjálmsson (Vite, vite, disait l'oiseau, 1958) ne cessent de la mettre en scène, ingénument, uniment, tout comme c'est elle qui dicta toute l'œuvre du plus grand poète islandais de ce siècle, Steinn Steinarr le nihiliste (Le Temps et l'Eau, 1948). Quand le Suédois Werner Aspenstrom, dans Théâtre III (1966) présente une araignée géante qui incarne l'avenir de notre espèce et symbolise notre inavouable penchant à l'auto-destruction, il se plie plus explicitement aux crochets d'une analyse qui fait de la Mort ainsi personnifiée l'âme même d'un certain type de fantastique, mais en général, dans le Nord, des affabulations aussi évidentes ne sont pas nécessaires. La Mort est au rendez-vous exact de tous les récits délibérément "fantastiques" de Karen Blixen : le mot même, chose rare en Scandinavie, figure expressément dans le titre danois (Sept récits fantastiques) qu'elle a donné à la version qu'elle proposa de ses Seven Gothic Tales (1934), initialement rédigés en anglais. Mais je considère que, précisément, cette variation GothicFantastisk est parfaitement représentative de la préoccupation que je cherche à illustrer. C'est certainement parce que le mot "gothique " aurait aussitôt suscité des réflexes d'ordre historique chez ses compatriotes que la grande Danoise n'a pas jugé bon de le reprendre dans sa propre langue. Ce qui mérite l'attention, c'est qu'elle avait d'abord songé à " gothique ", tant, dans un réflexe que Tolkien n'eût pas désavoué, il allait de soi que le fantastique, en soi, ne coïncidait pas, exprissis verbis, avec le but qu'elle visait.

6) Voilà pourquoi les «vampires, c'est-à-dire les morts qui s'assurent une perpétuelle jeunesse en suçant le sang des vivants " sont très rares en tant que tels dans les lettres scandinaves, tout comme on commence à s'en rendre compte au point où nous en sommes, toutes les manifestations trop ostentatoires ou démonstratives ou conventionnelles du fantastique classé. Ce n'est que par inférence que le thème du 
Vampire - de la femme-vampire, plus exactement - se rencontre chez Strindberg (ou dans les rêves de Swedenborg, tels qu'il les a consignés dans son Livre des rêves). Le personnage lui-même n'intervient guère, ou alors, seulement dans des textes mineurs. Ce qui vaut pour le vampire, motif classé et, comme le note R. Caillois, d'une indéniable monotonie, s'entend également pour les monstres, par lesquels j'ai entamé ces considérations: on en rencontre extrêmement peu dans les lettres scandinaves et, alors, à peu près toujours sous forme allégorique quand ce n'est pas par caractérisations vagues, comme ces monstres symboliques qui hantent L'île des Condamnés de Stig Dagerman (1946) où ils ne figurent qu'abstraitement, en quelque sorte, pour illustrer l'image de la condition humaine. En vérité, même quand un Norvégien comme Knud Faldbakken, dans Mauvaise année (1974-1976) cherche à figurer la damnation de l'homme actuel, les limites de son imagination fantastique ne vont pas au delà de la représentation de notre monde sous les espèces d'une décharge publique où doivent essayer de survivre quelques rescapés lamentables de la déchéance générale, mais de monstres ou d'images "fantastiques", point. Seulement, ou pour mieux dire, précisément, cette réalité trop bien connue, trop navrante aussi, de nos grandes villes, ainsi prise pour cadre de l'aventure humaine moderne, prend un relief et une force de conviction que n'atteignent pas les élucubrations futuristes ou " prospectives » dûment entérinées par un cinéma américain de complaisance.

7) Le motif suivant concerne "la statue, le mannequin, l'armure, l'automate qui, soudain, s'animent et acquièrent une redoutable indépendance »: on pense aussitôt à La Venus d'Ille de Mérimée, bien entendu.

14 Ce point me paraît intéressant, mais a contrario! Compte tenu, évidemment, des lacunes de mes connaissances, force m'est de dire que je ne connais aucun exemple de ce cas dans le Nord scandinave. Ce n'est pas qu'il soit totalement absent de certains récits de Karen Blixen, dans ce qu'elle appelle ses Contes d'Albondocani, par exemple, mais pas directement, pas ouvertement, uniquement par référence. J'en dirai autant de toute la thématique de don Juan et, donc, de l'image de la statue du Commandeur : ici comme partout, ailleurs, Kierkegaard en tête, encore une fois, don Juan a hanté les esprits du Nord et nous le retrouvons, plutôt à titre de sujet de méditations, chez Lars Gyllensten (Dans l'Ombre de don Juan, 1945). Mais pas de statue du Commandeur. Après tout, on peut s'en étonner : les sagas islandaises nous offrent parfois, rarement il est vrai, l'image de l'idole profanée d'un dieu qui s'anime et peut devenir meurtrière ; il est même difficile de décider si, dans Quand nous nous réveillerons d'entre les morts (1899) d'Ibsen, la statue du sculpteur Rubeck, qui est le véritable personnage de la pièce, existe en tant que telle ou, sous forme incarnée, sous les espèces de l'héroïne Maya, tout comme Strindberg s'est si bien appliqué, dans Un jeu de rêve (Le Songe, 1902) à donner à son œuvre une allure onirique qu'il ne nous est pas possible de savoir si la fille d'Indra est « réelle » ou non. Mais enfin, je ne vois pas d'équivalent, dans le Nord, du Golem d' Isabelle d'Egypte ou du mannequin de Marie de Melük-Blainville, tous deux d'Achim von Arnim. Pourtant, ouvertes comme elles l'ont toujours été aux influences étrangères, sensibles au rayonnement des grands chefs-d'œuvre consacrés en d'autre pays, il serait étrange que ces littératures n'eussent pas eu connaissance de textes qui connurent une telle audience ailleurs: il n'est que de voir, pour prendre un cas récent, comment Garcia Marquez ou Umberto Eco se retrouvent dans un des textes majeurs que nous ait donnés la littérature suédoise actuelle, avec le Gheel de Per Odensten (1981). Donc, force modèles ou archétypes fantastiques u' ont pas pu ne pas être connus du Nord. 
15 Mais justement : s'ils sont connus et, éventuellement, admirés, c'est uniquement par référence. Comme si une certaine dose de démesure ou une transgression trop marquée des bornes de la réalité répugnaient à ces esprits foncièrement pragmatiques et ennemis du délire. C'est un point sur lequel il faudra revenir. Contentons-nous, pour le moment, de le noter ici.

16 8) En revanche, « la malédiction d'un sorcier, qui entraîne une maladie épouvantable et surnaturelle » devrait être un lieu commun puisque c'est un élément presque banal de toute saga islandaise qui se respecte (voyez notamment Eyrbyggja saga, Grettis saga, Egils saga Skallagrímssonar ou, à titre d'épisodes secondaires, quantité de passages de Vatnsdoela saga). Or, le type se rencontre à peine dans les littératures modernes: on le pressent vaguement derrière certains épisodes du Cinquième hiver du magnétiseur de P.O. Enquist (1964), tout comme les sorcières hantaient Le Corsaire de la Baltique (1858) de Viktor Rydberg, mais l'indigence de nos sources, là encore, est éclairante.

Rydberg, d'ailleurs, nous tend une clef que nous aurions pu saisir bien plus tôt dans ce petit essai. Passé un temps d'affabulations plus ou moins complaisantes, il s'est très vite concentré sur ce qui, à l'évidence, correspondait mieux à ses goûts profonds: la relation de mythes et leur éventuelle interprétation, dans le sens de ses convictions et de son savoir. Un mythe, c'est d'abord, étymologiquement, une histoire à raconter, et, des sagnamenn à Lars Gustafsson en passant par Hjalmar Bergman, Olav Duun ou H.-C. Branner, jamais Scandinave n'a résisté à cette envie. D'autre part, immergé qu'il était dans la magie active, l'esprit du Nord n'a jamais cessé de quêter les correspondances secrètes, les liens occultes (Les liens invisibles, disait Selma Lagerlöf en 1894) qui nous rattachent à l'Autre Monde ou qui s'établissent entre vivants, et dont le sorcier est en quelque sorte l'orchestrateur attitré. Il reste que, même dans des œuvres particulièrement troubles, comme celle du Norvégien Ola Wiker, une certaine prise de distance de l'auteur à l'égard de son sujet et de ses personnages, ou un réflexe d'objectivation qui le pousse à chasser l'occulte dans des domaines classés (régionalisme, par exemple) interdisent de parler de propos fantastique délibéré. Ces inspirations ne consentent pas à l'outrance, elles veulent bien admettre l'anormal puisque, malheureusement, il est bien présent dans notre univers, mais, si j'ose dire, jamais par consentement aveugle. Le succès surprenant de Marx et de Freud dans le Nord, à l'évidence parce que l'un et l'autre fournissent une explication, dont on ne jugera pas, ici, la valeur, de phénomènes irrationnels ressortissant au sociologique ou au psychologique, n'a pas d'autre justification, il me semble.

9) «La femme-fantôme, issue de l'au-delà, séductrice et mortelle » pourrait renvoyer au personnage du draugr entrevu plus haut, sous 4). Elle aussi, au demeurant, appartient au fond commun de toutes nos littératures occidentales où elle sévit, telle quelle ou métamorphosée en animal (la renarde des contes chinois). Elle est familière de l'univers de H.-C. Andersen comme de celui de Z. Topelius, tous les folklores scandinaves la connaissent sous diverses dénominations et il y a fort à parier que c'est elle, l'énigmatique Úa de Kristnihald undir Jökli de H.K. Laxness (1969). Mais, au risque de me répéter, je redirai qu'elle ne paraît guère « fantastique » à nos yeux. Le même Laxness, par exemple, dans cette merveille qu'est Lumière du Monde (1937-1940) s'applique à percer les secrets de l'ineffable amour humain en en développant le visage à travers celui de toutes les amantes d'ólafur Ljósvikingur le scalde. On me comprend : ce qui est fantastique aux yeux du Prix Nobel islandais, ce n'est pas La Femme (avec des majuscules) mais le phénomène de l'amour ainsi constamment diversifié: cela, 
exactement, passe l'entendement et le poète, avec son intuition souveraine, s'attache à recomposer, à travers ses mille avatars, ce visage qui est "la beauté du monde ». Propos exactement "poḯtique " mais qui n'a de fantastique que sa tentative de décollage de la plate réalité, non son exclusion des possibles. Je pourrais aussi bien remonter à Seraphitus-Seraphita de Swedenborg, à Amandus-Amanda de Stagnelius et, au delà d'eux, à la présence obsédante des Dioscures dans la mythologie ancienne, c'està-dire, en somme, à la permanence dans les lettres scandinaves, notamment suédoises, de l'androgyne (pensons à la ou au Tintomara de C.J.L.Almquist). Ces cœurs scandinaves incorrigiblement épris de raison, de clarté, d'intelligibilité ont tout fait depuis presque trois millénaires et demi (pensons aux gravures rupestres de l'âge de bronze et à la paire alternative héliophore cheval/ bateau, traduite plus tard, vraisemblablement, par les jumeaux divins Freyr / Freyja ou par les nombreuses paires contrastives Ullr / Ullinn, Ódr / Ódinn, etc...) pour apprivoiser l'ineffable. Le propos est, en soi, fantastique puisqu'il entend outrepasser les limites du réel, sinon du possible, mais les résultats n'ont rien de terrifiant pour autant.

19 10) C'est pourquoi nous nous retrouvons en sol moins instable dans le dixième point de R. Caillois qui, pour le résumer, s'applique à « l'interversion des domaines du rêve et de la réalité ", celle-ci se dissolvant, disparaissant pour "laisser à sa place le songe [acquérir] l'écrasante solidité de la matière ». On ne saurait mieux cerner l'ensemble du "théâtre de rêve" de Strindberg avec ses flous, son apparente illogique, ses dédoublements, métamorphoses et substitutions, son animation consciente de ce que l'ou a appelé les «fantômes du moi » du personnage central, c'est-à-dire, derrière lui, de l'auteur. J'ai dit que les assises de ce procédé étaient fort antiques puisqu'il est comme banal, dans les eddas ou les sagas, de voir le héros passer d'un registre à l'autre, consciemment ou non, à telle enseigne que le lecteur ne sait plus toujours sur quel plan il évolue. Je ne parle pas tellement de l'importance que le rêve prend à titre épisodique dans tant d'œuvres actuelles (voyez, par exemple, à partir d'un procédé bien connu, la dissolution du dialogue ou l'ambiguité congénitale de la relation du témoignage dans toute l'œuvre du Suédois P.-O. Sundman), pas même de l'intention bien arrêtée, chez plus d'un conteur, de brouiller les pistes et de nous laisser ignorer où nous sommes : technique que nous trouvons admirablement orchestrée chez tant d'auteurs qui se sont intéressés, par priorité, aux marginaux, aux laissés pour compte de la société, aux vagabonds, dans le sillage de F. Jaeger ou de Kunt Hamsun, et qui tend à nous donner une réalité pour une autre, plus exactement, à substituer un fictif au prétendu réel ; on ne fera pas mieux, sous cet angle, que le personnage qui s'exprime à la première personne dans Faim (1890) de Knut Hamsun, mais on retrouve la même attitude dans nombre d'ouvrages plus récents, comme, pour ne prendre qu'un cas, dans les nouvelles ou le roman Une fille nu-tête (1961) du Norvégien Finn Bjørnseth. Le Danois Sven Holm a poussé le procédé plus loin encore, et sur le plan de la technique pure, dans ses écrits symboliques du genre Rex (1969) où il propose toute une série de schèmes utilisables à volonté au gré du lecteur : comme si l'argument initial n'avait d'autre fonction que de mettre en branle la rêverie selon des voies propres à chacun de nous, entreprise, en somme, assez identique au but que visait, en poésie, Raymond Queneau quand il nous proposait des millions de sonnets à partir d'une incitation première ré-arrangeable à volonté. Cela, en définitive, peut à la limite relever du jeu abstrait et gratuit. Mais je songe aussi à la technique, achèvement de la formule, qui consiste à inverser délibérément les signes en nous donnant par principe, mais sans le dire ouvertement, le rêve pour la réalité, ou inversement: beaucoup de romans que l'on qualifiera 
rapidement de surréalistes de l'Islandais Thór Vilhjálmsson pratiquent ce jeu de substitutions (ainsi Le Croissant de lune, 1976), le Danois Svend Åge Madsen poussant jusqu'au bout les procédés évoqués il y a un instant à propos de son compatriote Sven Holm, quand il rédige des « recueils » (Rajouts, 1967) publiés par fascicules dont on peut, à volonté, intervertir l'ordre.

Un point aura tout de même retenu l'attention, qui n'a plus le mérite de l'originalité dans ces pages-ci : une manière de réflexe d'expérimentateur préside à toutes ces tentatives. Nous ne sommes pas chez Julio Cortazar, l'impossibilité de distinguer ce qui est rêve et ce qui serait réalité n'a pas pour conséquence d'introduire une horreur qui viendrait de ce qu'à son insu, le lecteur a fini par prendre pour vrai alors qu'il ne s'agissait que d'un cauchemar. Là encore, les auteurs refusent d'oblitérer résolument toute ligne de démarcation. Il est presque amusant de conclure à une attitude d'intellectuels qui ne se décident pas à être dupes de leur affabulation chez des écrivains que l'on qualifie si allègrement de mystiques, visionnaires ou chimériques !

2111 et 12) C'est pour cela que je ne crois pas devoir retenir les deux derniers cas proposés par Roger Caillois: «la chambre, l'appartement, l'étage, la maison, la rue effacés de l'espace", comme dans La Ruelle ténébreuse de Jean Ray, et "l'arrêt ou la répétition du temps ", comme chez Potocki. Là encore, compte tenu, une fois de plus, de mes ignorances, j'ai vainement cherché, dans le Nord, des exemples d'abolition de nos catégories spatio-temporelles. Car c'est toucher là aux œuvres vives, c'est dépasser - dirai-je : à l'excès? - les possibilités d'une imagination qui a besoin, malgré toutes les fantaisies, de points d'ancrage sûrs dans le réel.

Le lecteur trouvera peut-être un peu décevantes les réflexions qui viennent de lui être proposées. Non, décidément, le Nord n'est pas fantastique, au moins dans les acceptions les plus débridées de ce dernier terme. Et plusieurs explications possibles de ce fait ont été suggérées dans les lignes qui précèdent. Je pressens bien que de telles affirmations ne manqueront pas de susciter l'étonnement, voire l'incrédulité. Et l'on n'omettra pas de m'accabler de noms d'auteurs et de titres qui témoignent d'un mode d'approche de la réalité assez peu réductible à nos habitudes mentales. Soit. Mais étrangeté, mystique, légende, rêverie vague, jeux sur le langage ne sont pas nécessairement fantastique dans les termes qui ont été posés en commençant.

C'est que le Nord est moins « abstrait » que nous - il est significatif que la seule école moderne qui se réclame de recherches purement abstraites en matière de littérature soit danoise (Ulla Ryum, Svend Åge Madsen, Cecil Bødker, qui ont tous écrit dans ces vingt dernières années), c'est-à-dire originaire de cette partie de la Scandinavie qui, depuis toujours, est " continentale ", donc plus proche de nos mentalités. Mais les jeux gratuits de l'intellect ne sont pas sincèrement pris au sérieux, ils demeurent des jeux que supplantent immanquablement les réalités quotidiennes, la petite maison de bois peinte, au fond d'un fjord ou au bord d'un lac, la lutte constante et assez fantastique, elle, contre un décor rude ou des éléments rebelles ou même l'engagement honnête dans des combats politiques et sociaux, pour le bienêtre, l'égalité et une certaine justice. Là, nous sommes en terrain stable: on ira jusqu'à dire qu'il y a effectivement quelque chose de fantastique dans le combat formidable que les prétendus 
"prolétaires" suédois, les hommes des revues Mot Dag, puis Profil, en Norvège, ont soutenu pour imposer ces idéaux qui viennent d'être rapidement énoncés. Mais évidemment, «fantastique » n'a plus, ici, le sens reçu depuis le début de cet article.

Méfiance de l'abstraction gratuite ou, pour exprimer une idée du même ordre en termes différents, répugnance à raffiner sur les excès de l'imagination. Il y a beau temps que l'on a fait remarquer à quel point l'imaginaire reste bien tempéré dans le Nord: il suffit de comparer n'importe quelle chanson de geste aux grands poèmes héroïques de l'Edda, ou mieux encore, les adaptations que firent les Islandais, au XIII ${ }^{\mathrm{e}}$ siècle, de nos romans courtois dans leurs sagas de chevaliers (riddarasögur). Un réalisme incoercible, un pragmatisme irréductible s'opposent, par nature, à toute outrance inconsidérée. On veut bien avoir peur à Copenhague, Reykjavik, Oslo ou Stockholm, mais pas gratuitement - ce qui ne préjuge pas, si l'on peut dire, de la qualité de la peur en question. Donc, préférons le roman-document, parce qu'il a de solides assises dans un concret vérifiable (et il fait flores là-bas, témoins P.-O. Enquist ou Sara Lidman, Suédois, Henrik Stangerup ou Thorkild Hansen, Danois, Jan Kjaerstad ou J. Michelet, Norvégiens, ou un mémorialiste qui s'entend si bien à romancer ses souvenirs comme l'islandais Jón Óskar) à toutes les élucubrations nées de cerveaux infiévrés. Le comble de ce qu'a pu enfanter de fantastique un génie comme Strindberg, c'est d'inverser les signes du rêve et de la réalité, mais les supports sensoriels n'en ont pas vraiment été affectés. Sinon, c'est le roman historique qui cristallise les pouvoirs d'un imaginaire ainsi limité par principe - et il se porte fort bien dans le Nord. Ou alors, l'allégorique vient à point nommé réduire les excès auxquels serait, d'aventure, portée la fiction: exemplaires, à cet égard, les Contes pour enfants de l'Islandaise Svava Jakobsdóttir ${ }^{10}$ : même une lecture rapide enseigne comment en dédramatiser l'horrible au prix d'une interprétation sociologique ou idéologique transparente.

Ce n'est pas, encore une fois, que les Scandinaves ne sachent s'entendre à faire jouer les ressorts d'un fantastique classé, mais le résultat est sans commune mesure avec nos délires latino-américains. Je pense ici aux célèbres "absurdistes" danois, Villy Sørensen, Peter Seeberg ou Leif Panduro ${ }^{11}$ Avec quelque effort, on pourrait faire entrer leurs nouvelles ou leurs romans dans quelques-unes des catégories qui viennent d'être proposées. Mais la conclusion serait toujours la même : nous avons à faire là à ce que j'appellerai un fantastique doux, à un fantastique marginal, sans prétention en tout cas parce qu'il finit par revenir à l'illustration d'un apologue, d'une fable dont l'argument, quand bien même il satisferait aux tendances du modernisme, se lit dès le début. Autant, pour procéder à l'inverse, trouver fantastiques bon nombre de contes de H.C. Andersen : en leur essence, ils peuvent l'être, mais on aura bien de la peine à trouver terrifiants "La Cloche», "L'Ombre» ou "Le Sapin», bien que tous les éléments constitutifs du genre y soient en place. En fait, ici comme ailleurs, le merveilleux l'emporte sur le fantastique, ce dernier est comme apprivoisé, on ne dira jamais assez à quel point les littératures scandinaves restent proches de nous. Humaines.

Et puis... Mettons que les Scandinaves soient moins portés que d'autres sur la métaphysique et l'occultisme abscons. Pour qui vit ou a vécu là-bas, sous ces latitudes, est-ce vraiment étonnant? Qui n'a jamais vu un coucher de soleil sur le Snaefellsjökull (Islande), une tempête sur Tórshavn (Féroe), les cascades complètement figées par la glace de Stora Sjöfall (Suède), les Lofoten vues en plein été de Hamaróy (Norvège), ou Odense (Danemark) au printemps aura peut-être quelque peine à comprendre que là, 
les bonnes vieilles recettes, dûment codifiées, $\mathrm{du}$ fantastique ne sont pas indispensables. Ce sont la nature, la lumière qui se chargent de faire basculer la réalité dans le fantastique, le merveilleux, le surnaturel, appelez cela comme vous voudrez. Si bien qu'au terme de ces quelques réflexions, la meilleurs idée, je crois, que l'on puisse donner du fantastique dans le Nord s'exprime dans le recueil de nouvelles du Féroien William Heinesen, tout récemment traduit en français, fond et forme. Mais surtout dans le titre qu'il a choisi : La lumière enchantée.

\section{NOTES}

1. Les monstres dans la littérature allemande du Moyen Âge, I-III, Göppingen, Kümmerle Verlag, 1982.

2. Voir R. Boyer, «Hamr, Fylgja, Hugr. L'âme pour les anciens Scandinaves ", dans Heimdal, n³3, 1981, pp. 5-10, vues reprises et étoffées dans Le Monde du Double. La magie chez les anciens Scandinaves, Paris, Berg International, 1986, pp. 32 et sq.

3. Par exemple: P.G. Castex, Anthologie du conte fantastique français, Paris, 1963 ; H.P. Lovecraft, Épouvante et surnaturel en littérature, Paris, 1969 ; M. Schneider, La littérature fantastique en France, Paris, 1964.

4. Là-dessus R. Boyer, Le Christ des Barbares, Paris, Cerf, 1987.

5. Claude Lecouteux, Fantômes et revenants au Moyen Age, Paris, Imago, 1986, ou R. Boyer, «Le draugr dans la littérature scandinave ancienne" dans Actes du Congrès sur Le Merveilleux, Oslo, 1986, également: Michel Meslin (sous la direction de), Le Merveilleux, Paris, Bordas, 1984, en particulier pp. 24-29 et 178-182.

6. Tous ces textes sont, en traduction française, dans Sagas islandaises, Paris, Gallimard, Gallimard, Bibliothèque de la Pléiade, 1987.

7. Pär Lagerkvbist, Ames masquées. La noce, présentées et traduites par R. Boyer, Paris, GF $n^{\circ} 424$, pp. 1-48.

8. Comme toute chose morte est merveilleuse

Et indicible,

Une feuille morte et un homme

mort

Et le disque de la lune.

9. Voir le numéro spécial de Plein Chant 25 / 26, 1985, notamment pp. 5-10 et 21-42.

10. - On trouvera une traduction de l'un de ces contes dans le numéro spécial d'Europe, mars 1983 («Littérature islandaise »), pp. 98-104.

11. R. Boyer, «Visages de l'absurde dans la littérature danoise de l'après-guerre » dans Ideas and ldeologies in Scandinavian Literature since the First Workd War ed. by Sveinn Skorri Höskuldsson, Reykjavík, 1975, pp. 101-122, ou «Les absurdistes danois des années 50 et 60 » dans Absurde et renouveaux romanesques 1960-1980, textes réunis par J. Bessière, Paris, Lettres modernes, 1986, pp. 31-54. 


\section{RÉSUMÉS}

La question posée dans cet article est de savoir s'il existe vraiment, dans les lettres scandinaves modernes, un « fantastique » correspondant aux normes reçues, telles qu'elles sont exposées, par exemple, par Roger Caillois dont les définitions et l'essai de thématique raisonnée sont pris comme point de départ. La démonstration qui est faite en suivant, point par point, les « cas » de fantastique proposés par Caillois aboutit à la conclusion que les Scandinaves n'ont que très rarement pratiqué le fantastique selon les acceptations classées du genre, qu'ils sont au contraire toujours restés sur les marges du fantastique. Hormis quelques rubriques qui reviennent à une tendance, profonde dans le Nord, à ne pas faire de démarcation tranchée entre ici-bas et au-delà (pacte avec le Diable, revenants et fantômes, par exemple), l'indigence de ces littératures en matière d'intervention du surnaturel provoquant la terreur, est caractérisée. Ce n'est pas que la terreur soit absente de ces inspirations, mais elle s'exerce sur de tout autres plans et à partir d'incitations tout à fait différentes. L'auteur remonte volontiers aux sources noroises pour montrer que, dès les origines connues, les lettres scandinaves ont toujours préféré le merveilleux au fantastique, ou le visionnaire à l'horrible, ou encore l'allégorique appliqué à l'idéologie, au sociologique ou au psychologique, à l'épouvantable. Il conclut cet essai d'ethnopsychologie appliqué à un point précis de l'expression littéraire en soulignant que le réalisme, le pragmatisme foncier des Scandinaves n'est pas à son aise dans les outrances de l'imagination et qu'au demeurant, la grande lumière du Nord, avec son pouvoir transfigurateur se prête naturellement à un tout autre type de fantastique que celui qui est conventionnellement reçu en littérature.

Bei der in diesem Artikel aufgeworfenen Frage geht es darum, die moderne skandinavische Literatur auf den Begriff des Phantastischen hin zu untersuchen, wie er von Roger Caillois verwendet und im einleitenden Aufsatz als Ausgangspunkt definiert wordenist. Punkt für Punkt wird dieser vorgegebene Begriff auf seine Gültigkeit untersucht, wolbei der Autor zu dem Ergebnis komnlt, daß es in den skandinavischen, Län dern kaum literarische Werke gibt die den Kriterien des Phantastischen gidt, die entsprechen, sondern daß sie immer am Rande des Phantastischen bleiben. Abgesehen von einigen Titeln, die unter die im Norden Titeln recht häufig vorkommende Tendenz fallen, zwischen dem Diesseits und dem Jenseits nicht zu unterscheiden (z.B. pakt mit dem Teufel, Gespenster usw.), ist das kaum vorhandene Eingreifen eines Schrecken auslösenden Übernatürlichen für diese Literaturen kennzekhnend. Das heißt aber nicht, daß das entsetzen in dieser Texten fehlt, doch wird es auf einer ganz anderen Ebene sicht bar und hat endere Ausgangssituationen. Der Autor geht vorzugsweise auf die altnordischen Ursprünge zurück, um aufzuzeigen, daß in der skandinavischen Literatur seit ihren uns bekannt en Anfängen immer das Wunderbar dem Phantastischen, den Visionäre dem Endsehlichen oder aber das Allegimische im Berung auf das Ideologische, das Socialogische oder das Psychologische dem Schrecklichen vorgeragen worden ist immer das Wunderbare dem Phanta tischen, das Visionare dem Entzetzlichen oder aber das Ailegorische wenn manes auf das Ideologische das Soziologische oder das Psychologische dem Schrecklichen vorgezogen worden ist. Er unterstreicht am ende seines ethnopsychologischen Aufsatzcs über ein gattungsspezifisches Problem der Literatur, daß der realismus und der bodcnständige Pragmatismus der Skandinavier für die Ausschweifungen der Phantasie nicht besonders brauchbar ist, und daß sich das große Nordlicht mit seiner transfigurativen Macht für eine ganz andere Art des Phantastischen eignet als die in der Literatur definierte. 
AUTEUR

RÉGIS BOYER

Université de Paris IV-Sorbonne 